\title{
Transcriptomic Analysis Reveals Both Coxsackievirus B3 Woodruff and GD Strain Using Similar Key Genes to Induce FoxO Signaling Pathway Activation in HeLa Cells
}

Mi Liu

National Institute for Viral Disease Control and Prevention, Chinese Center for Disease Control and Prevention

\section{Qian Yang}

National Institute for Viral Disease Control and Prevention, Chinese Center for Disease Control and Prevention

Jun Han ( $\square$ hanjun_sci@163.com )

National Institute for Viral Disease Control and Prevention, Chinese Center for Disease Control and Prevention

\section{Research Article}

Keywords: Transcriptom, CVB3, signaling pathway

Posted Date: February 15th, 2021

DOl: https://doi.org/10.21203/rs.3.rs-193617/v1

License: (c) (i) This work is licensed under a Creative Commons Attribution 4.0 International License. Read Full License 


\section{Abstract}

Coxsackievirus B3 (CVB3) is the major cause of viral myocarditis in human worldwide. Various studies have investigated the viral infection and pathogenic mechanisms. However, the precise disease mechanism is still not clear. In this study, RNA-seq technology was used to compare the transcriptomic profile of virus infected HeLa cells to the controls in order to analysis the key genes of host virus interaction. Two CVB3 strains, CVB3 Woodruff and GD16-69-CVB3 strain were selected to figure out the common disease mechanisms of both experimental and clinical strains respectively. Increased expression of cell cycle genes CCNG2, GADD45B, PIM1, RBM15, KLF10 and RIOK3, down regulation of $C Y B A$ were detected. Autophagy genes $A T G 12$ and YOD1 were also upregulated during CVB3 infection. Slightly increase of SOD2 and $A T G 12$ were shown in the expression of infected cells, meanwhile, little change was detected in GABARAP expression. Further, FoxO signaling pathway was enriched by KEGG analysis, shown a close interaction with the DEGs in the PPI network. Genes of related pathways such as cell cycle, autophagy and oxidative stress resistance were confirmed by RT-PCR as well. In conclusion, our results reveal that FoxO signaling pathway is a common mechanism activated during the infection of both CVB3 strains. And this pathway plays a regulatory role in downstream pathways such as cell cycle, autophagy, oxidative stress resistance and antiviral immune responds.

\section{Introduction}

Coxsackievirus is a group of non-enveloped single-stranded RNA virus, one member of Picornaviridae family, Enterovirus genus, causing clinical symptoms ranging from respiratory illness and aseptic meningitis to acute and chronic myocarditis. Coxsackievirus B3 (CVB3) is the most common cause of viral myocarditis in children and young people. The cytopathology directly induced by virus infection and replication as well as the after activation of immune responds are two common causes of myocardia damages. However, the precise disease mechanism of CVB3 induced viral myocarditis is still not clearly demonstrated.

Numbers of clinical and experimental studies are about the disease mechanism and the signaling pathways involved ${ }^{1}$. One most studied step of CVB3 infection is invasion, and the binding of CVB3 to the host cell is crucial. It depends on the interaction between viral capsid protein and cell receptor CAR (Coxsackievirus, and adenovirus receptor) and the co-receptor DAF (decay-accelerating factor) also known as CD55. Once entering the host cell, CVB3 could manipulate the host system for its own replication ${ }^{2}$. The replication or virus life cycle results in disruption of host cellular process and signaling pathway events ${ }^{3,4}$.

It is well known that there are only a few CVB3 strains specific for laboratory research models such as CVB3 Nancy and Woodruff which were all developed decades ago in regions outside of China ${ }^{5}$. On the other hand, clinical isolations form patients are evolving with each of the outbreaks in a great diversity. In China, CVB3 outbreaks associated with hand, foot, and mouth disease (HFMD), and their sequences showed regional phylogenetic dynamics which reveals the connections between CVB3 outbreaks and 
evolution ${ }^{6}$. In this study, two CVB3 strains with distinctions between sequence and origin were selected, one is an experimental stain CVB3 WoodruffखCVB3-Wखand another is a Chinese epidemic strain GD16-69CVB3 $₫ C V B 3-G D \otimes$ from the 2016 outbreak, so that to further figure out their pathogenetic similarities and differences during infection. We use RNA-seq technology to analysis the transcriptomic profiles after the virus infection, aiming to gain a broader view of genes involved in the host virus interaction and greater insights of the common disease mechanisms causing by both clinical and experimental stains.

\section{Method}

Cells and virus. HeLa cells were cultured in Dulbecco's modified Eagle's medium (DMEM) supplemented with 10\% (v/v) Fetal Bovine Serum (FBS) (GIBCO, Thermofisher, Australia), 100 units/ml penicillin, and $100 \mu \mathrm{g} / \mathrm{ml}$ streptomycin. Coxsackievirus B3 Woodruff strain (CVB3-W) was performed in HeLa cells using standard methods. Coxsackievirus B3 strain GD16-69-CVB3 (CVB3-GD), (MT712317) was kindly provided by National Laboratory of Poliomyelitis, National Institute for Viral Disease Control and Prevention, China CDC.

CVB3 infections. CVB3-W strain ( $\mathrm{MOI}=10)$, and CVB3-GD strain ( $\mathrm{MOI}=7$ ) was propagated in HeLa cell at $37^{\circ} \mathrm{C}$. After inoculation, cells were washed twice with PBS and maintained in fresh DMEM ( $\left.2 \% \mathrm{FBS}\right) .2 \mathrm{~h}$, $4 \mathrm{~h}$ and $6 \mathrm{~h}$ later, infected cells and mock cells were harvested for transcriptome and quantitate RT-PCR analyses.

Western blot analysis. HeLa cells were lysed with buffer (Beyotime), then centrifuged at $13,000 \times \mathrm{g}$ for 10 min at $4^{\circ} \mathrm{C}$. Before boiling, the protein concentration was determined by the Enhanced BCA protein Assay kit (Thermo scientific). $30 \mu \mathrm{g}$ of protein of each sample was subjected to $10 \%$ SDS polyacrylamide gel electrophoresis and transferred to $0.02-\mathrm{mm} \mathrm{NC}$ membranes (GE health). After blocking with $5 \%$ nonfat milk for $1 \mathrm{~h}$ at room temperature, the membranes were incubated with VP1 (Dako) and Actin (Cell signaling) primary antibodies at $4^{\circ} \mathrm{C}$ overnight. After washing with PBST thrice, the membranes were incubated with horseradish peroxidase-conjugated secondary antibodies (Cell signaling) for $60 \mathrm{~min}$ followed by washing. Finally, protein bands were detected with chemiluminescence detection kit (PerkinElmer).

RNA isolation and next-generation sequencing of mRNA (RNA-Seq). At selected time points the total RNA was extracted using TRIzol Reagent (Sigma, USA) according to the manufacturer's protocol and the RNA quality was determined by RNA Nano 6000Assay Kit of the Bioanalyzer 2100 system (Agilent Technologies, CA, USA). The mean RIN value was 9.87 (0.83). The library preparation and RNA-Seq were performed by Novogene Co., Ltd, Beijing, China. A total amount of $1 \mu \mathrm{g}$ RNA per sample was used for library preparation. The sequences' clustering was performed on a cBot Cluster Generation System using TruSeq PE Cluster Kit v3-cBot-HS (Illumia) according to the manufacturer's instructions. The library preparations were sequenced on an Illumina Novaseq platform and 150 bp paired-end reads were generated. 
Data processing. Raw sequencing reads of fastQ format were processed through in-house perl scripts on the platform of Novogene Co., Ltd. with adaptors, poly-N containing reads and low-quality reads being removed. The Q20,30, and GC content and the clean data were calculated. All the downstream analyses were based on the clean data with high quality. The reads mapping to the reference human genome GRCh38/hg38 (GRCh38.p12) and gene model annotation files were downloaded from genome website ENSEMBL (ftp://ftp.ensembl.org/pub/release-94/assembly_chain/homo_sapiens/) directly. Hisat2 v2.0.5 was used to build the reference genome index for the paired-end clean reads alignment. FeatureCounts v1.5.0-p3 was used to count the gene level reads numbers mapped to each gene and calculated by number of uniquely mapped reads per kilobase per million mapped reads (RPKM).

Differential expression analysis. Differential expression analysis between two groups (virus infected group compared to control) was performed using the DESeq2 R package (1.20.0) based on a statistical model of negative binomial distribution. The resulting P-values were adjusted using the Benjamini and Hochberg's approach for controlling the false discovery rate. Genes with an adjusted P-value $<0.05$ and |log2Fold Changel $(|\log 2 \mathrm{FC}|)>0$ found by DESeq2 were assigned as differentially expressed genes (DEGs). Heat map diagram was constructed using pheatmap R package. Venn diagram was created using VennDiagram ${ }^{31}$ to show the quantitative distribution of DEGs in different comparisons. Integration of protein-protein interaction (PPI) network analysis of DEG was visualized using STRING website (https://string-db.org/).

GO and KEGG Enrichment analysis for DEGs. Gene Ontology (GO) enrichment analysis of DEGs was implemented by the clusterProfiler R package, in which gene length bias was corrected. GO terms with corrected P-value $<0.05$ were considered significantly enriched by DEGs. Pathway analysis was performed using the Kyoto Encyclopedia of Genes and Genomes (KEGG) database ${ }^{32}$. Cluster Profiler R package also used to test the statistical enrichment of differential expression genes in KEGG pathways.

Quantitative RT-PCR. Total RNA was extracted from cells using TRIzol Reagent (Sigma, USA). Eighteen host genes were selected for real-time quantitative PCR (RT-qPCR) analysis. Primers for target genes analysis were listed in Additional file S1. First strand cDNA synthesis was performed using PrimeScript RT reagent Kit (TaKaRa, Japan). qRT-PCR detection was conducted using TB Green ${ }^{\circledR}$ Premix Ex Taq ${ }^{\text {TM }}$ II (TaKaRa, Japan) and LightCycler ${ }^{\circledR} 480$ System (Roche Applied Science) following the manufacturer's recommendations. The quantitive RT-PCR conditions were as follows: $95^{\circ} \mathrm{C}$ for $30 \mathrm{~s}$, followed by $5 \mathrm{~s}$ at $95^{\circ} \mathrm{C}, 30 \mathrm{~s}$ at $60^{\circ} \mathrm{C}$ for 40 cycles. GADPH gene was used as internal control. The primers used were in Table S1. The mRNA relative expression ratios of the treated group versus that of the control group were calculated by the $2^{-\triangle \Delta C T}$ method $^{33}$.

Statistical Analysis. The statistical comparisons between the control and infected groups were performed using an unpaired two-tailed Student's t test or a Mann Whitney test (Graphpad prism Software, La Jolla, CA, USA) and $p<0.05$ was considered statistically significant.

\section{Results}


Identification of CVB3 dynamic replication. To identify the virus replication, HeLa cells were infected with CVB3-W (MOI=10) and CVB3-GD strain (MOI=7) Ørespectively for 2, 4 and 6h. The expression of CVB3 VP1 protein was examination by Western blot. The expression of VP1 protein of two strains increased gradually as time prolonged from $2 \mathrm{~h}$ post-infection (pi) to 6hpi (Figure $1 \mathrm{~A}, \mathrm{~B}$ ). VP1 of two strains showed similar expression trend (Figure 1). Therefore, the titer of two virus strains was suitable to compare the transcriptomic changes.

DEG analysis. To further study the cellular damage mechanism of CVB3 infection, transcriptomic changes of HeLa cells infected with two CVB3 strains were analyzed at $2 \mathrm{~h}, 4 \mathrm{~h}$ and $6 \mathrm{~h}$ pi by RNASequencing respectively. Comparison was made between CVB3-W or CVB3-GD infected HeLa cells and the control cells using three-dimensional PAC (Principal Component Analysis), result reveals that the samples were distinctly distributed as mock control samples and two different virus strains infected samples in a time dependent manner (Figure 2A). In the differential expression analysis, a gene with an adjusted P-value $<0.05$ and |log2Fold Change| $>0$ were considered as DEGs. There were 137 (69 upregulated and 68 downregulated DEGs), 1432 (926 upregulated and 506 downregulated DEGs) and 2790 DEGs (1879 upregulated and 911 downregulated DEGs) changed significantly at 2, 4 and $6 \mathrm{~h}$ after CVB3-W infection respectively. Meanwhile, 1702 (805 upregulated and 896 downregulated DEGs), 1188 (773 upregulated and 415 downregulated DEGs) and 4338 (2748 upregulated and 1590 downregulated DEGs) significantly changed DEGs at 2, 4 and $6 \mathrm{~h}$ after CVB3-GD infection respectively. Compared to control HeLa cells, the relative numbers of common DEGs in CVB3-W and CVB3-GD infected samples of all time points were 73 (55 upregulated and 18 downregulated DEGs) and 396 (236 upregulated and 160 downregulated DEGs) respectively. The up and down regulation of these DEGs were uniquely displayed in the heatmaps (Figure 2B, 2C). Then, GO and KEGG analysis were performed with the common DEGs induced by each of the two CVB3 strains. The GO enrichment of the CVB3-W infection induced 73 common DEGs, shown significance of pathways such as cellular responds of peptide and extracellular stimulus, involving cellular components of cell division and adhesion junctions, and the molecular functions related to transcriptional regulation and signaling transduction (Figure S1 A). Meanwhile, the KEGG enrichment results suggested that infection, inflamation pathways, MAPK signaling and autophagy related pathways were more stimulated compared with control samples (Figure S1 B). After that we found that 396 CVB3-GD infection associated DEGs enriched a different set of pathways. In the GO enrichment result, nuclear exporting and chromosomal regional cellular processes, muscle and cardiac ventricle development pathways, actin filament activity and ribosomal protein binding functions were significantly enriched (Figure S1 C). Moreover, pathways such as cell cycle, FoxO signaling and viral myocarditis involved pathways were significantly enriched in the KEGG pathways, and immune responds and infection related pathways were also with significance as in the results of CVB3-W infected DEGs (Figure S1 D).

To gain more similarity in the pathways involved in both viral strains infection, KEGG pathway enrichment analysis of infected samples of all three time points with each virus strain was performed. As in the Figure 2D, the top enriched pathways associated with CVB3-W infection induced DEGs were cell cycle, FoxO signaling pathway, RNA transport, endocytosis, mismatch repair and ubiquitin mediated proteolysis. 
And in CVB3-GD infected samples, DEGs were associated with cell cycle, proteoglycans in cancer, longevity regulating pathway, human T-cell leukemia virus 1 infection, RNA transport and FoxO signaling pathway in the KEGG pathway enrichment analysis (Figure 2E). These results reveal that the common pathways of these two virus strains infection are cell cycle, RNA transport, and FoxO signaling pathway. Among them FoxO signaling pathway could be stimulated by both strains and function as the upstream regulator of other DEGs associated enrichment pathways ${ }^{7,8}$.

Identical DEGs of HeLa cells infected with CVB3. To investigate the identical DEGs changes induced from CVB3-W and CVB3-GD strain, all DEGs were compared each other at 2, 4 and 6hpi. As shown in the Venn diagram, 58 common DEGs induced by two strains were found in three time points (Figure 3). Of these 58 differentially expressed genes, 43 were upregulated after infection and 15 were downregulated (Table S2). GO and KEGG enrichment analysis also performed to further conclude the mechanisms associated with both viral strains' infection. The results revealed that those identical genes were involved in similar cellular pathways that consistent with the results enriched individually. GO enriched terms such as cellular responds of peptide and extracellular stimulus, cell division and adhesion junctions involving processes and transcriptional regulation were with significance. Signaling transduction and viral myocarditis, autopahgy and immune responds related pathways were mainly listed in the KEGG enrichment (Figure 2D, 2E, S1). To be more specific, many genes of those 58 DEGs were involved in the pathways coincide with the enrichment results and also downstream of FoxO signaling. For example, UBE2S, PIM1 and KLF10 were factors in cell cycle regulation ${ }^{9-11}$, PHAX and RBM27 were all RNA binding proteins and related to RNA transportation ${ }^{12,13}$.

The protein-protein interaction network of DEGs. FoxO proteins are a group of transcription factors playing essential regulatory roles in gene expression of cellular processes such as apoptosis, cell-cycle progression and oxidative stress resistance. To identify the inner reason of pathway regulatory and key DEGs interaction, genes from the 58 common DEGs, the most enriched KEGG pathways and FoxO signaling pathway were selected for PPI network analysis using STRING website (Figure 4A). The nodes represent DEGs and pathway proteins with specific colors corresponding to the expression levels. Among them, all four types of FoxO genes (FoxO1, FoxO3, FoxO4 and FoxO6) were strongly interacted with each other in the network shown the essential role of the FoxO signaling pathway. The KEGG pathways of FoxO signaling shown in Figure 4B clearly present the pathway regulation correlated with DEGs of CVB3W and CVB3-GD strain infected samples vs. control cells. Both CVB3 strains induced common DEGs in HeLa cells were associated with FoxO signaling pathways, upregulated EDN1, RIOK3, PIM1, KLF1O, RBM15, OTUD3, YOD1, XPO1 and down regulated CYBA.

Transcriptome confirmation and pathway protein verification using qRT-PCR. To confirm the RNA-seq result, qRT-PCR was used to analysis the expression level of selected DEGs. Among the 58 common DEGs, eight upregulated and one downregulated DEGs were selected for qRT-PCR analysis. Consist with the transcriptome results, both CVB3-W and CVB3-GD infection could induce a statistically increase in mRNA expression levels of XPO1, EDN1, RIOK3, PIM1, KLF10, RBM15, OTUD3 and YOD1, a decrease of CYBA in HeLa cells compared to controls at 2, 4 and 6hpi (Figure 5B, C). Meanwhile, the expression of 
nine FoxO signaling proteins selected from protein-protein interaction network (PPI) analysis were verified at the same time to reveal the significance of CVB3 infection on pathway regulation. In accordance with RNA-seq and the PPI results, FoxO1 (Figure 5A), CCNG2, ATG12, GADD45B and SOD2 were upregulated (Figure 5B, C), whereas FoxO6 (Figure 5A) expression was downregulated gradually at 2, 4 and 6hpi.

\section{Discussion}

In this study, two CVB3 strains were selected. A novel Coxsackievirus strain CVB3-GD was selected from Chinese outbreak in 2016 as an epidemic strain compared with a conventional experimental strain CVB3$W$ to investigate viral pathogenesis. Except for their regional evolutionary differences, the specific sequence pattern in the virus-host interface is also noticeable. The study of Pan et al. found that the substitution of single amino acid residues of CVB3 VP3 Q243E and VP2 D138N could attenuated the binding of CVB3 and the host DAF receptor ${ }^{14}$. With VP3-234Q and VP2 $138 \mathrm{~N}$ on the capsid protein of CVB3-W, and VP3-243E and VP2-138D on the host interaction surface protein side of CVB3-GD, during virus invasion, both strains were loosely bind the host DAF receptors based on different molecular basis. To better understand the viral pathological effect on cells, we first confirmed that the collected cells were in the state of gradual proliferation of virus by VP1 antibody's in the WB (Figure 1). Subsequently, transcriptomics analysis of CVB3 infected HeLa cells was performed using RNA-seq of the Novogene platform, especially to identify genes associated with host-virus interaction, cellular signaling pathways activation and immune responds. Previous studies have demonstrated several mechanisms in CVB3 infected HeLa cells such as cell death, immune respond and autophagy ${ }^{15,16}$. Our RNA-Seq results reveal that the infection of both virus strains could stimulate several cellular pathways such as cell cycle, apoptosis and RNA transport and all together result in the enrichment of FoxO signaling pathway (Figure 2D, 2E), highlight this pathway as a common mechanism in both the experimental and epidemic CVB3 strains induced disease progression.

FoxO proteins are a subgroup of the Forkhead family of transcription factors characterized by a conserved DNA-binding domain (the "Forkhead box"), which are crucial gene expression regulators in apoptosis, cell-cycle progression and oxidative stress resistance. All these cellular functions, could contribute to its important role in the host virus interaction in innate immunity. During Sendai virus infection, FoxO1 acts as a negative regulator of RIG-I-triggered signaling, promotes virus replication and down regulates type I IFN production ${ }^{18}$. In the study of Japanese encephalitis virus, the infection results in a decrease in FoxO protein expression, induces apoptosis by inhibiting STAT3-Foxo-Bcl-6/p21 pathway ${ }^{19}$. In our result, EDN1 (Endothelin-1) was one of the upregulated common DEGs after CVB3 infection, which encoded a peptide survival factor in colon cancer, directly regulated by FoxO1 and NF-к $\beta$ pathway (León et al. (2014)). In this study, the qRT-PCR results of both CVB3 strains' infection leads to FoxO1 upregulation and FoxO6 downregulation, while had little effect on FoxO3 and FoxO4 expression (Figure 5A). This indicated the FoxO protein involved innative immunity regulation and antiviral mechanism could also function in the process of CVB3 infection. 
Furthermore, our RNA-seq data shown a significant importance of alternative expression patterns of HeLa cells after CVB3 infection. Combined with KEGG pathway enrichment results, the selected DEGs are closely related to cell cycle, apoptosis, autophagy, oxidative stress, DNA repair and immune responds, which are regulated by upstream FoxO signaling pathway ${ }^{17}$. First, cell cycle was one of the most significantly enriched KEGG pathways down stream of FoxO proteins. RT-PCR results shown an upregulation of FoxO-cell cycle signaling proteins CCNG2 and GADD45B. Meanwhile, four cell cycle related DEGs: PIM1, RBM15,KLF10 and RIOK3 were also upregulated. PIM1 is a serine/threonine-protein kinase involved in cell cycle and cell survival, the active PIM1 could phosphorylate PRAS40 which binds with pFox03a, finally down regulates apoptosis ${ }^{20}$. And RBM15 (RNA binding motif protein 15) is crucial in cell survival, cell cycle progression, has an apoptosis inhibition effect in CML cells ${ }^{21}$. The multifunctional roles of those genes confirmed the close interaction between cell cycle and apoptosis regulation in CVB3 infection. The KLF10 (Krueppel-like factor 10), also known as Tieg1, regulates gene transcription of multiple pathways involving proliferation, apoptosis, and cell cycle arrest. The study of TIEG knocked out mouse model demonstrated its role in cardiac hypertrophy ${ }^{11}$, therefore $K L F 10$ might be one potential target gene in CVB3 induced cardiac pathology. RIOK3 is also a serine/threonine-protein kinase activating type I IFN pathway by bridging TBK1 and IRF3, plays a critical role in innate immune responds against both DNA and RNA virus ${ }^{22}$, which is a presumable target gene with CVB3 antiviral function. Secondly, autophagy is another signaling pathway that regulated by FoxO signaling. Studies already reveal that CVB3 could benefit from autophagosome and autophagic flux for its own replication ${ }^{23}$. Unlike cell cycle proteins, the RT-PCR results revealed autophagy gene ATG12 is only slightly up regulated during infection, until 6hpi, only CVB3-GD infected samples shown this significance, in the meanwhile there was not any obvious expression changes of GABARAP during the infection. Accordingly, the autophagy related DEG XPO1 shown small expression changes during infection. XPO1 (Exportin-1) is a nuclear exporting protein could be phosphorylated by STK38, plays an important role in autophagy regulator BCEN1 exit ${ }^{24}$. In a recent published study, XPO1 proved to be a perfect antiviral target for virus access blocking ${ }^{25}$, which could be inferred as a potential protective gene against CVB3 invasion. The expression of YOD1 is significantly upregulated in RNA-seq and RT-PCR result after CVB3 infection. Its deubiquitin enzymic function makes it a necessary component to clear damaged lysosomes and promote autophagosome formation ${ }^{26}$. Those results brought different aspects to investigate the connection between host autophagy and CVB3 infection. In PPI network, the only DEG directly connected with YOD1 is OTUD3, another deubiquitinating enzyme up regulated by CVB3 infection, which is important in several cellular process such as cell cycle. Resent study discovered that, RNA virus infection could stimulate OTUD3 deacetylation by SIRT1, shut down its deubiquitinate function, release MAV (mitochondrial antiviral-signaling protein) and finally promote the quick propagation of innate immune signaling of host antiviral defense ${ }^{27}$. So that a similar antiviral function in pathological mechanism during CVB3 infection was further speculated. Thirdly, oxidative stress and DNA repair is also a signaling pathway that stimulated by CVB3 infection under the regulation of FoxO signaling. As confirmed in RT-PCR results $G A D D 45 B$ was up regulated during infection and $S O D 2$ is slightly up regulate until $6 \mathrm{hpi}$. The DEG $C Y B A$ encoded protein is a crucial subunit of NADPH oxidase involve in oxidative stress, and researchers have 
studied its role in HCV and HBV disease mechanism ${ }^{28,29}$. In both RNA-seq and RT-PCR result, CYBA was down regulated during virus infection. The study of HIV shown that HIV Nef could act on the components of NADPH oxidase complex, therefore alters the superoxide production in neutrophils and modulate the innate defense mechanism ${ }^{30}$. We could predict similar mechanisms in CVB3 infection. Altogether, many of the genes in our study not only belong to the pathways under the regulation of FoxO signaling pathway as a member, but also have their own roles in different cellular process which are valuable targets for antiviral treatment.

\section{Conclusion}

In this study, RNA-seq technology is used to compare the transcriptomic profiles of HeLa cells infected with two different CVB3 strains, one is a widely used research strain CVB3-W, the other is a Chinese epidemic strain CVB3-GD. We reveal that no matter how the subtle molecular basis and regional evolution changes, FoxO signaling pathway is the common disease mechanism of these two strains of CVB3. Also, KEGG enrichment and PPI network of DEGs indicate the regulatory features of FoxO proteins and their down-stream pathways such as cell cycle, apoptosis, autophagy, oxidative stress, DNA repair responds and antiviral immune responds. We encourage our colleagues to further investigate the inner connections of those key genes and signaling pathways, to discover deeper insights of the disease mechanism of CVB3 induced viral myocarditis.

\section{Declarations}

\section{Acknowledgements}

We appreciate kind support from Wenbo Xu, the director of National Institute for Viral Disease Control and Prevention, Chinese Center for Disease Control and Prevention, for the resources and knowledge of CVB3 epidemic viruses.

Author contributions statement

M.L and J.H. conceived, designed the experiments and revised the manuscript. M.L. performed the experiments, analyzed the data and wrote the manuscript. Q.Y. contributed reagents and materials. As the correspondence of this study, J.H. had the access to all the data in the study and takes responsibility for the integrity of the data and the accuracy of the data analysis.

\section{Additional information}

Supplementary information accompanies this paper at

Competing Interests: The authors declare no competing interests.

\section{References}


1. Garmaroudi, F. S. et al. Coxsackievirus B3 replication and pathogenesis. Future Microbiol. 10, 629653 https://doi.org/10.2217/fmb.15.5 (2015).

2. Peischard, S., Ho, H. T., Theiss, C., Strutz-Seebohm, N. \& Seebohm, G. A. Kidnapping Story: How Coxsackievirus B3 and Its Host Cell Interact. Cell Physiol Biochem. 53, 121-140 https://doi.org/10.33594/000000125 (2019).

3. Wang, Y. et al. The Capsid Protein VP1 of Coxsackievirus B Induces Cell Cycle Arrest by UpRegulating Heat Shock Protein 70. Front Microbiol. 10, 1633 https://doi.org/10.3389/fmicb.2019.01633 (2019).

4. Luo, X. N. et al. Coxsackievirus B3 Infection Triggers Autophagy through 3 Pathways of Endoplasmic Reticulum Stress. Biomed Environ Sci. 31, 867-875 https://doi.org/10.3967/bes2018.115 (2018).

5. Knowlton, K. U., Jeon, E. S., Berkley, N., Wessely, R. \& Huber, S. A mutation in the puff region of VP2 attenuates the myocarditic phenotype of an infectious cDNA of the Woodruff variant of coxsackievirus B3. J Virol. 70, 7811-7818 https://doi.org/10.1128/jvi.70.11.7811-7818.1996 (1996).

6. Han, Z. et al. Two Coxsackievirus B3 outbreaks associated with hand, foot, and mouth disease in China and the evolutionary history worldwide. BMC Infect Dis. 19, 466 https://doi.org/10.1186/s12879-019-4107-z (2019).

7. Huang, H. \& Tindall, D. J. Dynamic FoxO transcription factors. J Cell Sci. 120, 2479-2487 https://doi.org/10.1242/jcs.001222 (2007).

8. Cheng, Z. The FoxO-Autophagy Axis in Health and Disease. Trends Endocrinol Metab. 30, 658-671 https://doi.org/10.1016/j.tem.2019.07.009 (2019).

9. Williamson, A. et al. Identification of a physiological E2 module for the human anaphase-promoting complex. Proc Natl Acad Sci U S A. 106, 18213-18218 https://doi.org/10.1073/pnas.0907887106 (2009).

10. ladevaia, V. et al. PIM1 kinase is destabilized by ribosomal stress causing inhibition of cell cycle progression. Oncogene. 29, 5490-5499 https://doi.org/10.1038/onc.2010.279 (2010).

11. Rajamannan, N. M. et al. TGFbeta inducible early gene-1 (TIEG1) and cardiac hypertrophy: Discovery and characterization of a novel signaling pathway. J Cell Biochem. 100, 315-325 https://doi.org/10.1002/jcb.21049 (2007).

12. Machitani, M., Taniguchi, I., McCloskey, A., Suzuki, T. \& Ohno, M. The RNA transport factor PHAX is required for proper histone $\mathrm{H} 2 \mathrm{AX}$ expression and DNA damage response. Rna. 26, 1716-1725 https://doi.org/10.1261/rna.074625.120 (2020).

13. Silla, T. et al. The human ZC3H3 and RBM26/27 proteins are critical for PAXT-mediated nuclear RNA decay. Nucleic Acids Res. 48, 2518-2530 https://doi.org/10.1093/nar/gkz1238 (2020).

14. Pan, J. et al. Single amino acid changes in the virus capsid permit coxsackievirus B3 to bind decayaccelerating factor. J Virol. 85, 7436-7443 https://doi.org/10.1128/jvi.00503-11 (2011).

15. Jensen, K. J. et al. An ERK-p38 subnetwork coordinates host cell apoptosis and necrosis during coxsackievirus B3 infection. Cell Host Microbe. 13, 67-76 https://doi.org/10.1016/j.chom.2012.11.009 (2013). 
16. Esfandiarei, M. et al. Coxsackievirus B3 activates nuclear factor kappa B transcription factor via a phosphatidylinositol-3 kinase/protein kinase B-dependent pathway to improve host cell viability. Cell Microbiol. 9, 2358-2371 https://doi.org/10.1111/j.1462-5822.2007.00964.x (2007).

17. Accili, D. \& Arden, K. C. FoxOs at the crossroads of cellular metabolism, differentiation, and transformation. Cell. 117, 421-426 https://doi.org/10.1016/s0092-8674(04)00452-0 (2004).

18. Ma, Z. et al. Forkhead box 01-mediated ubiquitination suppresses RIG-I-mediated antiviral immune responses. Int Immunopharmacol. 107152, https://doi.org/10.1016/j.intimp.2020.107152 (2020).

19. Guo, F. et al. Japanese encephalitis virus induces apoptosis by inhibiting Foxo signaling pathway. Vet. Microbiol. 220, 73-82 https://doi.org/10.1016/j.vetmic.2018.05.008 (2018).

20. Kim, W. et al. PIM1-activated PRAS40 regulates radioresistance in non-small cell lung cancer cells through interplay with FOXO3a, 14-3-3 and protein phosphatases. Radiat Res. 176, 539-552 https://doi.org/10.1667/rr2609.1 (2011).

21. Yang, Y., Wang, S., Zhang, Y. \& Zhu, X. Biological effects of decreasing RBM15 on chronic myelogenous leukemia cells. Leuk. Lymphoma. 53, 2237-2244 https://doi.org/10.3109/10428194.2012.684350 (2012).

22. Feng, J. et al. RIOK3 is an adaptor protein required for IRF3-mediated antiviral type I interferon production. J Virol. 88, 7987-7997 https://doi.org/10.1128/jvi.00643-14 (2014).

23. Shi, X. et al. Coxsackievirus B3 infection induces autophagic flux, and autophagosomes are critical for efficient viral replication. Arch Virol. 161, 2197-2205 https://doi.org/10.1007/s00705-016-2896-6 (2016).

24. Martin, A. P. et al. STK38 kinase acts as XPO1 gatekeeper regulating the nuclear export of autophagy proteins and other cargoes. EMBO Rep. 20, e48150 https://doi.org/10.15252/embr.201948150 (2019).

25. Uddin, M. H., Zonder, J. A. \& Azmi, A. S. Exportin 1 inhibition as antiviral therapy. Drug Discov Today. https://doi.org/10.1016/j.drudis.2020.06.014 (2020).

26. Papadopoulos, C. et al. VCP/p97 cooperates with YOD1, UBXD1 and PLAA to drive clearance of ruptured lysosomes by autophagy. Embo j. 36, 135-150 https://doi.org/10.15252/embj.201695148 (2017).

27. Zhang, Z. et al. Acetylation-Dependent Deubiquitinase OTUD3 Controls MAVS Activation in Innate Antiviral Immunity. Mol Cel/ 79, 304-319 e307, doi:10.1016/j.molcel.2020.06.020 (2020).

28. Li, Q. et al. Integrative functional genomics of hepatitis $C$ virus infection identifies host dependencies in complete viral replication cycle. PLoS Pathog. 10, e1004163 https://doi.org/10.1371/journal.ppat.1004163 (2014).

29. Ma, N. et al. Oxidative Stress-Related Gene Polymorphisms Are Associated With Hepatitis B VirusInduced Liver Disease in the Northern Chinese Han Population. Front Genet. 10, 1290 https://doi.org/10.3389/fgene.2019.01290 (2019).

30. Salmen, S. et al. HIV-1 Nef associates with p22-phox, a component of the NADPH oxidase protein complex. Cell Immunol. 263, 166-171 https://doi.org/10.1016/j.cellimm.2010.03.012 (2010). 
31. Chen, H. \& Boutros, P. C. VennDiagram: a package for the generation of highly-customizable Venn and Euler diagrams in R. BMC Bioinformatics. 12, 35 https://doi.org/10.1186/1471-2105-12-35 (2011).

32. Kanehisa, M. et al. KEGG for linking genomes to life and the environment. Nucleic Acids Res. 36, D480-484 https://doi.org/10.1093/nar/gkm882 (2008).

33. Livak, K. J. \& Schmittgen, T. D. Analysis of relative gene expression data using real-time quantitative PCR and the 2(-Delta Delta C(T)) Method. Methods. 25, 402-408 https://doi.org/10.1006/meth.2001.1262 (2001).

\section{Figures}
A
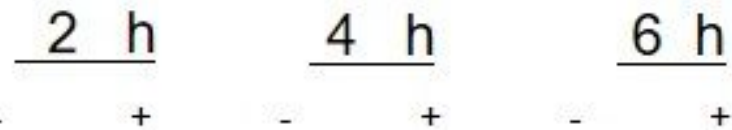
CVB3-W $\mathrm{MOI}=10$
$32 \mathrm{kDa}$
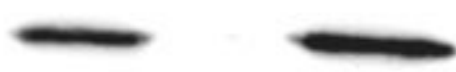
VP1
$42 \mathrm{k} \Gamma^{-}$
Actin
B
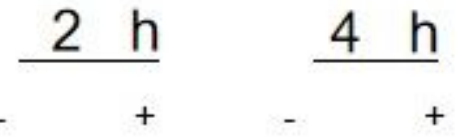
$\frac{6 \mathrm{~h}}{+}$
CVB3-GD $\mathrm{MOI}=7$
$32 \mathrm{kDa}$
VP1

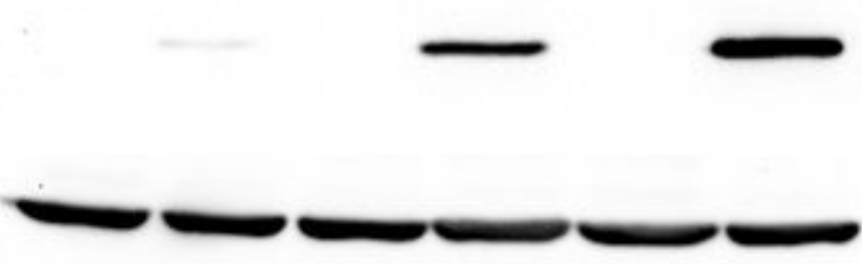

$42 \mathrm{kDa}$

Actin

\section{Figure 1}

Western blot results of CVB3 VP1 and Actin protein expression levels in CVB3 infected and uninfected HeLa cells. (A) Western blot detection of VP1 and Actin proteins expressed in HeLa cells infected with CVB3-W strain ( $\mathrm{MOI}=10)$ for indicated time points. In each time point, the lane "-" represented uninfected 
control cells and "+" represented CVB3-W infected cells. (B) Western blot detection of VP1 and Actin proteins expressed in HeLa cells infected with CVB3-GD strain ( $\mathrm{MOI}=7)$ for indicated time points. In each time point, the lane "-" represented uninfected control cells and "+" represented the results of CVB3-GD infected cells. The complete blot graph were in Figure S3.
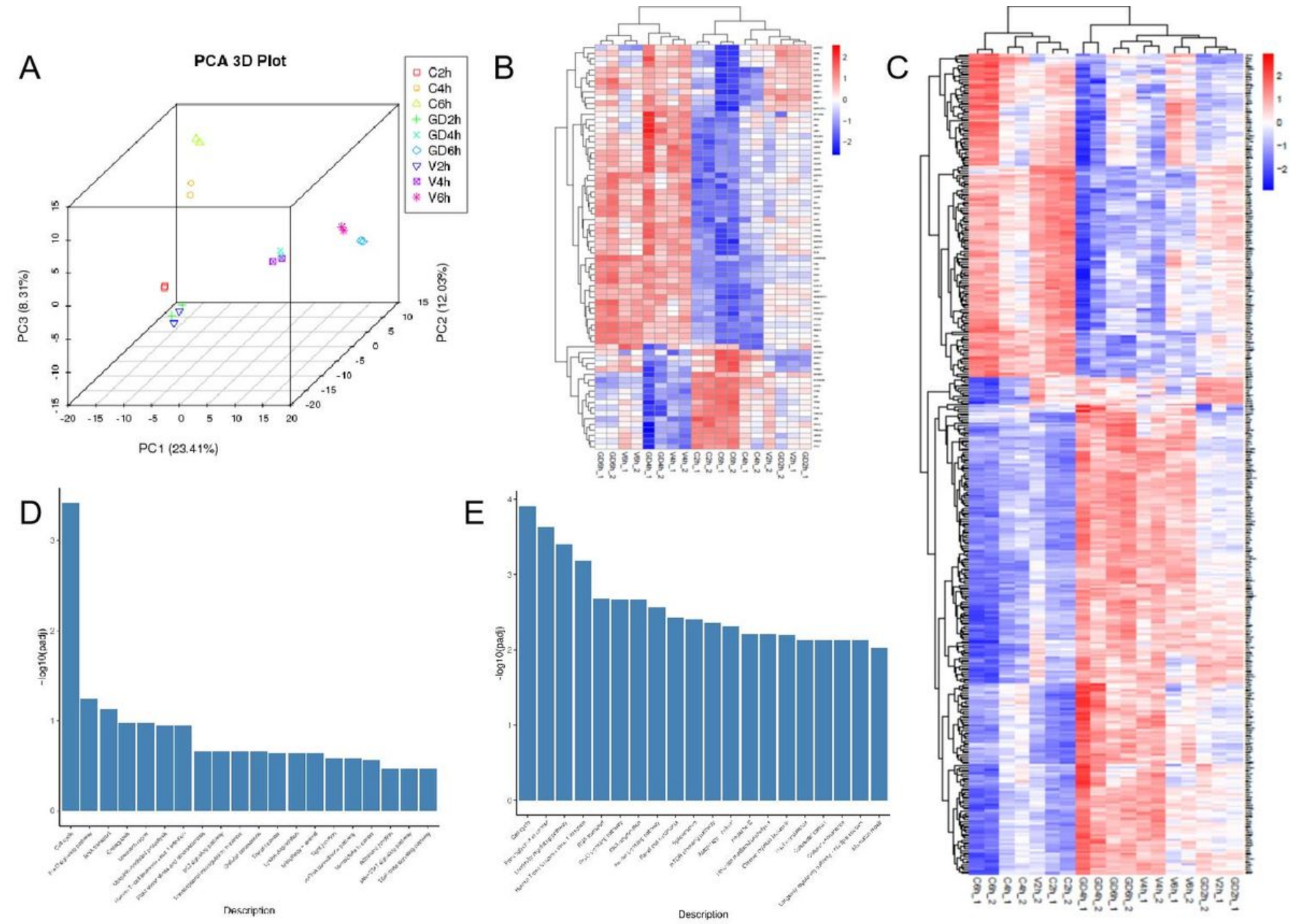

Figure 2

Differential expression (DE) of the transcript expression profile of HeLa cells infected with two CVB3 strains compared to HeLa control cells. (A) Principal component analysis of HeLa cell transcripts from three different culture conditions (C, control HeLa cells 2, 4, 6hpi; V, HeLa cells infected with CVB3-W at 2, 4, 6hpi and GD, HeLa cells infected with CVB3-GD at 2, 4, 6hpi). Each group were represented in the color codes upper right of the plot. (B) Heat map of 73 DEGs of CVB3-W infected HeLa cells compared with controls. Log2 fold change (-2 to 2) values are represented in four colors shade. (C) Heat map of 396 DEGs of CVB3-GD infected HeLa cells compared with controls. Log2 fold change (-2 to 2 ) values are represented in four colors shade. (D) Plot of the top 20 KEGG pathways enriched in CVB3-W infected HeLa cells compared with controls. (E) Plot of the top 20 KEGG pathways enriched in CVB3-GD infected HeLa cells compared with controls. 


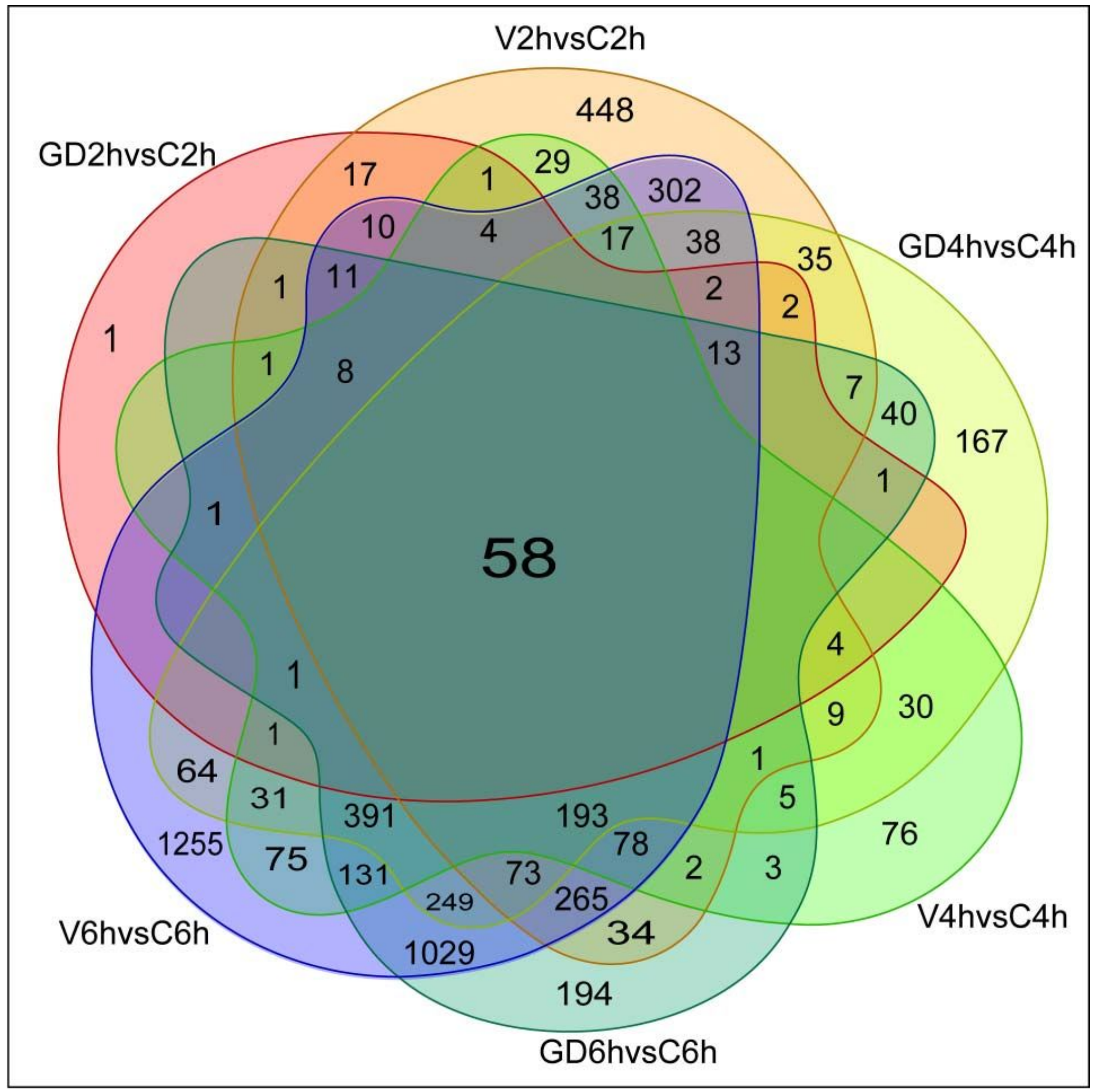

Figure 3

Venn diagrams of DEGs transcripts. The numbers in each area represents the number of DEGs among various combinations (V2h vs $C 2 h$, V4h vs $C 4 h$, V6h vs $C 6 h, G D 2 h$ vs $C 2 h, G D 4 h$ vs $C 4 h$ and GD6h vs C6h). The overlapping area in the diagrams represents common DEGs between combinations. 

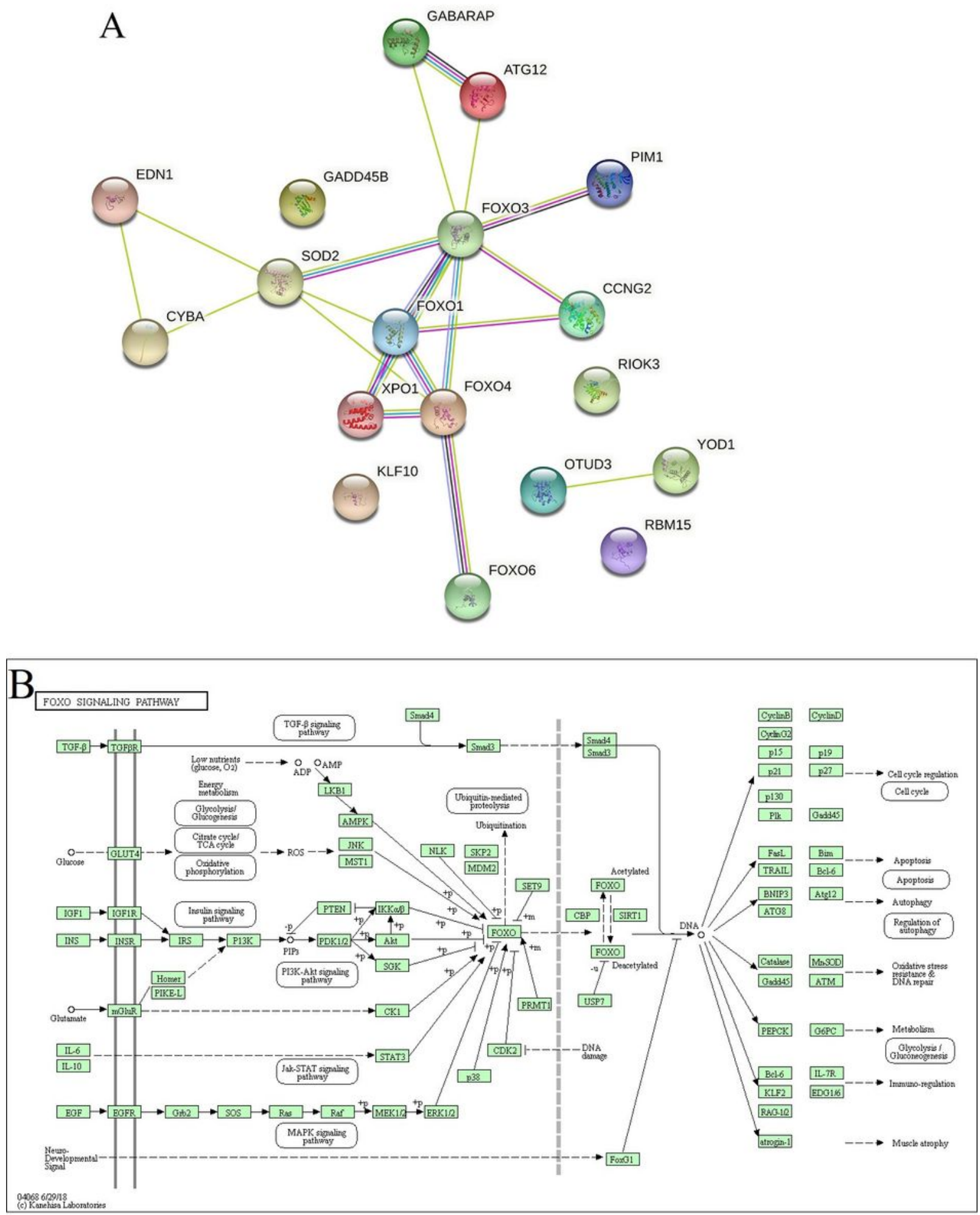

\section{Figure 4}

Comprehensive analysis of gene expression profiles of two CVB3 strains infected HeLa cells shown the importance of FoxO signaling pathway. (A) The protein-protein interaction (PPI) network analysis in STRING with 18 protein that differently expressed during both CVB3 strains' infected HeLa cells compared with controls. (B) FoxO signaling pathway from KEGG database, with several DEGs in diagram (A), shown the regulatory association of pathways downstream. 

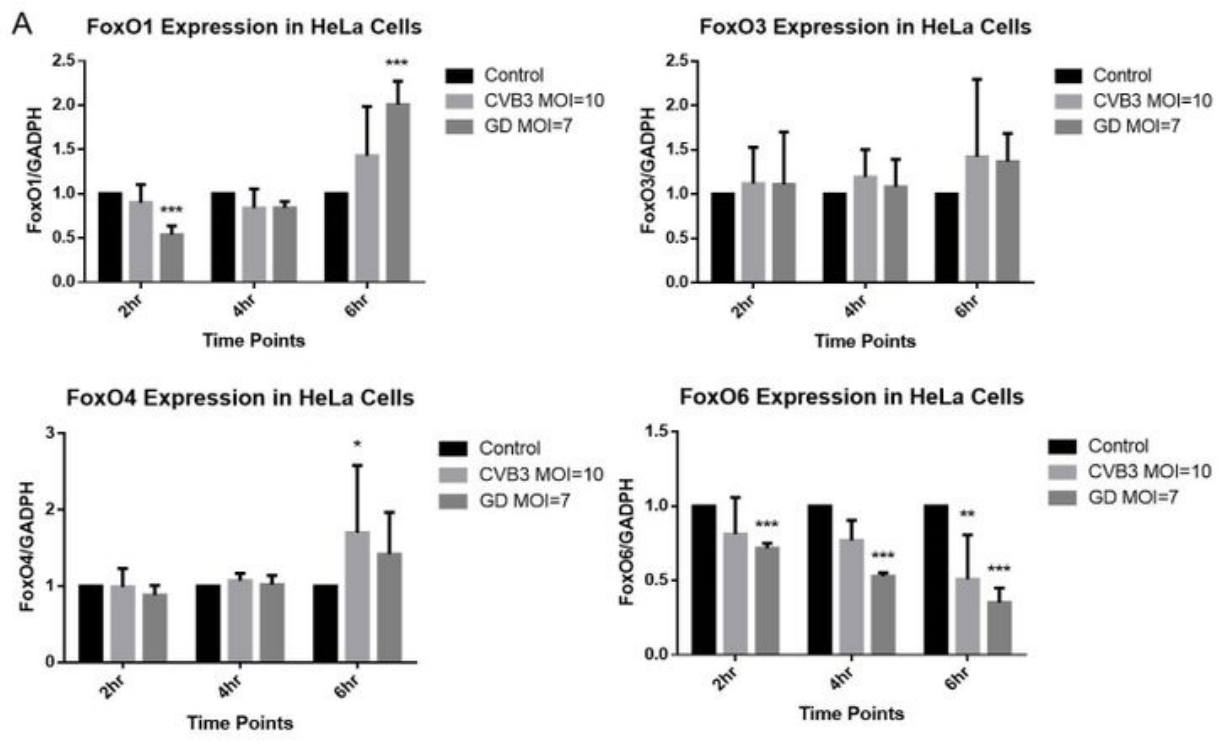

B
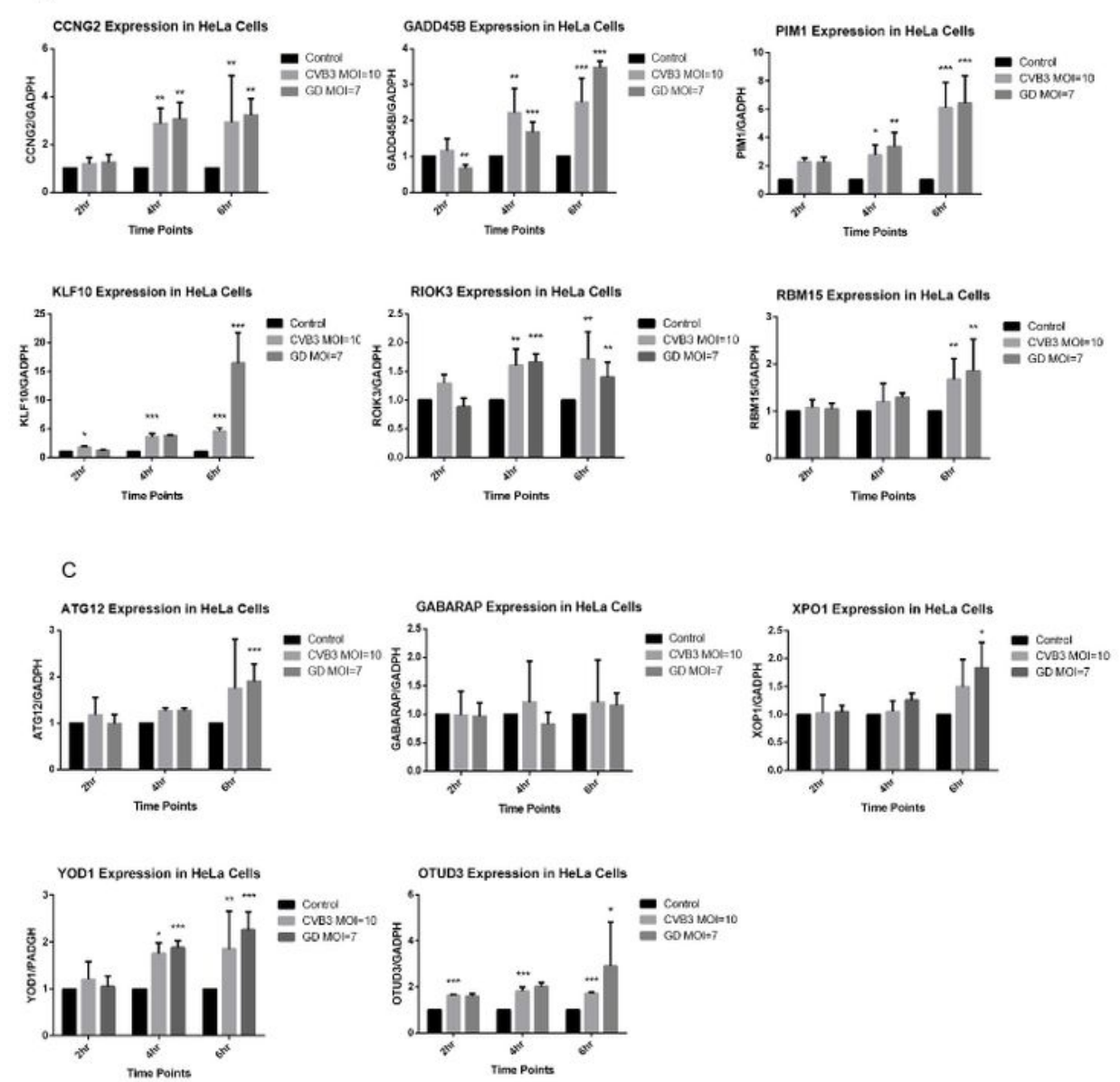

Figure 5

Quantitative RT-PCR analysis of 18 selected genes from 58 DEGs and FoxO signaling pathway. HeLa cells were infected with CVB3-W (MOI=10) or CVB3-GD strain ( $\mathrm{MOI}=7)$. The transcript levels of each gene were compared between CVB3 infected and non-infected control HeLa cells, ${ }^{\star} p<0.05,{ }^{\star \star} p<<0.01,{ }^{\star \star \star} p<0.001$.

\section{Supplementary Files}


This is a list of supplementary files associated with this preprint. Click to download.

- SupplementaryFiles.doc

- Tables2.xls 